\title{
Evaluation of GSTP1 and APC methylation as indicators for repeat biopsy in a high-risk cohort of men with negative initial prostate biopsies
}

\author{
Bruce J. Trock ${ }^{1}$, Michelle J. Brotzman ${ }^{1,}$, Leslie A. Mangold ${ }^{1}$, Joseph W. Bigley ${ }^{2}$, Jonathan \\ I. Epstein ${ }^{3}$, David McLeod ${ }^{4}$, Eric A. Klein ${ }^{5}$, J. Stephen Jones ${ }^{5}$, Songbai Wang ${ }^{6}$, Theresa \\ McAskill $^{2, \dagger}$, Jyoti Mehrotra ${ }^{6}$, Bhargavi Raghavan ${ }^{6}$, and Alan W. Partin ${ }^{1}$ \\ ${ }^{1}$ Brady Urological Institute, Johns Hopkins School of Medicine, Baltimore, MD \\ ${ }^{2} \mathrm{MDxHealth}$, Durham, NC \\ ${ }^{3}$ Department of Pathology, Johns Hopkins School of Medicine, Baltimore, MD \\ ${ }^{4}$ Department of Urology, Walter Reed Army Medical Center, Washington, DC \\ ${ }^{5}$ Glickman Urological and Kidney Institute, Cleveland Clinic, Cleveland, $\mathrm{OH}$ \\ ${ }^{6}$ Ortho-Clinical Diagnostics, Johnson \& Johnson, Raritan, NJ, USA
}

\begin{abstract}
- To evaluate the performance of DNA methylation biomarkers in the setting of repeat biopsy in men with an initially negative prostate biopsy but a high index of suspicion for missed prostate cancer.

- We prospectively evaluated 86 men with an initial histologically negative prostate biopsy and high-risk features.

- All men underwent repeat 12-core ultrasonography-guided biopsy.

- DNA methylation of glutathione-S-transferase P1 (GSTP1) and adenomatous polyposis coli (APC) was determined using tissue from the initially negative biopsy and compared with histology of the repeat biopsy.

- The primary outcome was the relative negative predictive value (NPV) of APC compared with GSTP1, and its $95 \%$ confidence interval (CI).

- On repeat biopsy, 21/86 (24\%) men had prostate cancer.

- $\quad$ APC and GSTP1 methylation ratios below the threshold (predicting no cancer) produced a NPV of 0.96 and 0.80 , respectively. The relative NPV was 1.2 (95\% CI: 1.06-1.36), indicating APC has significantly higher NPV.

- Methylation ratios above the threshold yielded a sensitivity of 0.95 for APC and 0.43 for GSTP1.
\end{abstract}

\footnotetext{
Correspondence: Bruce J. Trock, Brady Urological Institute, Johns Hopkins School of Medicine, 600 N. Wolfe St., Baltimore, MD 21287, USA. btrock@jhmi.edu.

*Current affiliation: Westat, Inc., Rockville, MD, USA.

†Current affiliation: self-employed.
}

Supporting information Additional Supporting Information may be found in the online version of this article under the DOI reference: 
- Combining both methylation markers produced a performance similar to that of APC alone.

- APC methylation patterns were consistent with a possible field effect or occurrence early in carcinogenesis.

- APC methylation provided a very high NPV with a low percentage of false-negatives, in the first prospective study to evaluate performance of DNA methylation markers in a clinical cohort of men undergoing repeat biopsy.

- The potential of APC methylation to reduce unnecessary repeat biopsies warrants validation in a larger prospective cohort.

\section{Keywords}

prostate cancer; biopsy; methylation; APC; GSTP1

\section{INTRODUCTION}

The limited specificity of PSA [1,2] and the false-negative rate of prostate biopsy schema $[3,4]$ result in large numbers of men with elevated PSA and/or other high-risk features but an initial histologically negative prostate biopsy. Many of these men continue to undergo biopsy procedures even though only 10-36\% of second biopsies detect cancer [5-9], and cancer detection decreases after each subsequent negative biopsy [10]. Improved methods are needed to identify men who can forgo a repeat biopsy, particularly if the trigger for initial biopsy or the initial biopsy histology indicate high-risk features. The emphasis, therefore, is on achieving a high negative predictive value (NPV) and a low false-negative rate. In initial studies of prostate cancer, DNA methylation markers have been found to have high sensitivity and specificity [11-13]. We conducted a prospective study in a clinical cohort to provide a preliminary estimate of the NPV of DNA methylation markers in men whose initial biopsy was negative but for whom there was a high index of suspicion for a missed cancer.

\section{PATIENTS AND METHODS}

\section{Study design}

The study protocol specifically targeted three groups considered to have a high index of suspicion of prostate cancer after a histologically negative initial biopsy: Group 1: suspicious DRE or high-risk PSA level (PSA $\geq 8.0 \mathrm{ng} / \mathrm{mL}$ and prostate volume $<50.0 \mathrm{cc}$, PSA density $\geq 0.2 \mathrm{ng} / \mathrm{mL} / \mathrm{cc}$, or per cent free PSA (\%fPSA) $\leq 10.0$ ); Group 2: high-grade prostatic intraepithelial neoplasia (HGPIN); Group 3: atypical small acinar proliferation (ASAP) on initial biopsy. These subgroups had a $20-60 \%$ probability of prostate cancer detection on repeat biopsy $[6-9,14]$, and were hypothesized a priori to exhibit different biomarker performance characteristics.

Consecutive men, whose initial biopsy was negative for cancer and who fell into one of the three risk groups, were enrolled from Johns Hopkins Hospital, Walter Reed Army Medical Center and the Cleveland Clinic, after institutional review board-approved protocols had been followed and informed consent obtained. These men either had undergone their initial biopsy at one of the three study centres, or had been referred to one of the study centres after a negative biopsy performed by an outside urologist. Other inclusion criteria included: age 40-75 years; no biopsy $\leq 12$ months before the initial (enrollment) biopsy (biopsies $>12$ months before initial biopsy were permitted); adequate paraffin-fixed biopsy tissue based on a minimum of sextant biopsy and sections totalling $\geq 50 \mu$; and agreeing to undergo 12-core 
repeat biopsy at the study centre as standard of care. Exclusion criteria included: previous history of cancer other than non-melanoma skin cancer; previous prostate or bladder surgery; previous rectal surgery precluding prostate biopsy; bleeding dyscrasia; use of anticoagulants within 5 days of study entry; previous BCG treatment; previous treatment with finasteride, dutasteride, LHRH analogues/antagonists, antiandrogen, oestrogen or PCSPES within 3 months of study entry; and >24 months elapsing between the initial enrolment biopsy and repeat biopsy.

Slides from paraffin-embedded fixed tissue from initial and repeat biopsies were sent to Johns Hopkins Hospital and read by a single blinded reader (J.I.E.), and tissue sections totaling $50 \mu$ from each biopsy were sent to Veridex, LLC for quantitative methylation testing, blinded to pathology outcome. To minimize the risk of missing cancer in tissue sections used for methylation testing, the blinded reader also examined haematoxylin and eosin sections that flanked the tested sections. Data were analysed at Johns Hopkins Hospital.

\section{Sodium bisulphite modification of DNA}

Tissue deparaffinization, lysis and sodium bisulphite modification of DNA were done as described previously [15]. Universally methylated (CpG-M) and unmethylated (CpG-U) genomic DNA (500 ng input) from Chemicon (Temecula, CA, USA) were used in PCR reactions as positive and negative DNA methylation controls, respectively, to generate standard curves for copy number determination.

\section{Quantitative methylation-specific PCR}

Probes and primers were obtained from Scandinavian Gene Synthesis AB (Köping, Sweden). Sequences are shown in Supplementary Table 1. Cycle threshold $(\mathrm{Ct})$ values for glutathione-S-transferase P1 (GSTP1) and adenomatous polyposis coli (APC) were determined in a single-gene PCR reaction on ABI 7900HT using FAM-labelled scorpion probes and Fast Start Taq DNA polymerase (Roche Molecular Diagnostics, Germany). The final primer probe concentrations were 250nM each for the two GSTP1 amplicons and $500 \mathrm{nM}$ each for APC primer probe. PCR cycling conditions were $95^{\circ} \mathrm{C}$ for $5 \mathrm{~min}$, followed by 40 cycles of $95^{\circ} \mathrm{C}$ for $30 \mathrm{~s}, 59^{\circ} \mathrm{C}$ for $30 \mathrm{~s}$ and $72^{\circ} \mathrm{C}$ for $30 \mathrm{~s}$, followed by a 5 -min extension at $72^{\circ} \mathrm{C}$. Using the same modified DNA samples, $\beta$-actin Cts were determined in a multiplex PCR using labelled scorpion probes GSTP1-FAM, APC-TxR and $\beta$-actin-Cy5 on Smart Cycler II (Cepheid, Sunnyvale, CA, USA) ${ }^{15}$. Owing to sample limitations, $\beta$-actin was not repeated on the ABI, but we had previously shown in an independent sample of 10 prostate cancers and 10 benign prostate tissues that both approaches gave similar $\beta$-actin Cts; Pearson correlation $=0.987$.

Standard curves were generated and the $\mathrm{Ct}$ value for each of the three genes was converted to a copy number represented as a methylation ratio ([methylated gene copies/ $\beta$-actin copies] $\times 1000$ ) to normalize for the amount of input DNA. Samples were classified as 'methylated,' 'unmethylated,' or 'no test' if methylation ratios were above the threshold, below the threshold with $>200 \beta$-actin copies, or $<200 \beta$-actin copies with no positive methylation signal, respectively. Methylated status was defined as methylation ratio $>1$ for GSTP1 and >2 for APC. The GSTP1 threshold was determined in previous feasibility studies of 672 biopsy and 140 prostatectomy specimens; this threshold gave consistent sensitivity and specificity with both specimen types. The APC threshold was adjusted in the present study to maximize overall sensitivity at an acceptable specificity ( $\geq 40 \%$ in Groups 1 and 2). 


\section{Statistical analysis}

Samples from the initial negative biopsy were classified as positive (methylated) or negative (unmethylated) based on GSTP1, APC, or the combined result (methylated, if either gene was methylated, vs. unmethylated, if neither gene was methylated). These results were compared with cancer status defined by the repeat biopsy histology, and biomarker performance was characterized by positive predictive values (PPVs) and NPVs, sensitivity, and specificity. The relative NPV (ratio of NPVs for two tests) and its 95\% CI were used to compare the NPV of APC with that of GSTP1. A 95\% CI with lower bound >1.0 suggests a significant advantage for the test represented in the numerator [16]. The McNemar chisquared test was used to compare sensitivity or specificity of the two biomarkers Continuous variables were compared using the $t$-test or Wilcoxon rank-sum test for normally distributed and non-normally distributed variables, respectively [17]. Receiver operating characteristic (ROC) curves were derived and compared using MedCalc v11.5 (MedCalc Software, Marienkerke, Belgium).

The present study was sponsored by Veridex, LLC, but was designed, conducted, analysed, and the manuscript prepared by investigators at the three participating academic centres.

\section{RESULTS}

Initially, 162 consecutive participants were prospectively enrolled. Of these, 40 were subsequently found not to meet eligibility criteria because they had previous cancer history $(n=3)$, did not meet the criteria for any of the three risk groups $(n=6)$, prostate cancer was not identified in a re-read of initial biopsy $(n=4)$, or there was $>24$ months between initial and repeat biopsy $(n=27)$. Thus, 122 eligible men were enrolled. Of these, $36(30 \%)$ could not be analysed owing to lack of a valid methylation assay result $(n=1)$, insufficient tissue from initial biopsy $(n=25)$, insufficient tissue from repeat biopsy $(n=1)$, or slides from initial biopsy being unavailable for re-read $(n=9)$. This left 86 eligible participants for the analysis cohort (70\% of eligible men).

Participants who were and were not included in the analysis cohort did not differ significantly with respect to age, race, body mass index, DRE result, PSA, \%fPSA, prostate volume, PSA density, risk group, or prevalence of prostate cancer (data not shown); however, data on PSA, \%fPSA, and prostate volume obtained within 1 year of initial biopsy were only available for 110, 64 and 85 participants, respectively. These measurements were not always available because many participants were referred from outside the study centres after a negative initial biopsy,

Characteristics of the analysis cohort are shown for each of the three risk groups in Table 1A. Cancer incidence (determined by repeat biopsy) was $24 \%$ (21/86); within Groups 1, 2 and 3 the incidence was $14 \%, 21 \%$, and $39 \%$, respectively.

The distribution of methylation ratios is shown graphically for GSTP1 and APC within each risk group, stratified by repeat biopsy result (cancer, no cancer) in Fig. 1. For APC (Fig. 1A) almost all values below the threshold were non-cancers, while for GSTP1 (Fig. 1B) there was more overlap. Geometric mean methylation ratios for both markers were consistently higher for cancer than no cancer, with the exception of GSTP1 in Group 1; these differences were significant for APC for all participants combined ( $P=0.012$ ), and for Group 2 (HGPIN; $P=0.032$ [Table 1B]). Both markers showed the highest methylation ratios in Groups 2 and 3 , consistent with higher risk of prostate cancer after initial diagnosis of either HGPIN or ASAP. 
The methylation patterns suggest possible links between APC and GSTP1. Among participants with cancer, APC methylation ratios were significantly higher for biopsies that also had GSTP1 methylation (28.37) compared with those without GSTP1 methylation (7.67), $P=0.002$. Also among participants with cancer, APC methylation often occurred in biopsies without concomitant GSTP1 methylation, but GSTP1 was only methylated in biopsies also exhibiting APC methylation.

Table 2 shows performance characteristics of GSTP1 and APC methylation. For all three risk groups combined, the NPV was 0.96 (95\% CI 0.81-1.0) for APC vs. 0.80 (95\% CI 0.68-0.89) for GSTP1; the relative NPV was 1.2 (95\% CI 1.06, 1.36), suggesting a significant advantage to APC. Sensitivity was 0.95 for APC vs. 0.43 for GSTP1 $(P<0.001)$. However, this came at the expense of a higher false-positive rate: 0.60 for APC vs. 0.25 for GSTP1 $(P<0.001)$. The area under the ROC curve was 0.62 (95\% CI 0.51-0.72) for GSTP1, and 0.68 (95\% CI $0.57-0.78)$ for APC; the difference was not significant $(P=0.355$ [Supplementary Fig. 1, Supplementary Table 2]).

We also considered the performance of both markers used in combination (Table 2C). The combination had the same performance as APC alone. Furthermore, as most urologists would re-biopsy a man with ASAP (Group 3), we also evaluated performance for just Groups 1 and 2 combined. This resulted in minimal changes in performance.

Because \%fPSA is often used to determine the need for biopsy we compared its performance with that of GSTP1 and APC. \%fPSA was available for 37 participants. Using a threshold of $15 \%$, \%fPSA yielded a sensitivity of 0.63 , specificity of 0.54 , and NPV of 0.83 . Among the same 37 participants, the corresponding values for APC were 1.0, 0.38 and 1.0 , while the GSTP1 values were $0.38,0,76$ and 0.82 .

\section{DISCUSSION}

We observed that the DNA methylation marker APC alone, or combined with GSTP1, resulted in an NPV of $96 \%$ in men with a high index of suspicion for cancer after an initial histologically negative biopsy, who subsequently underwent repeat biopsy. Although a high NPV was achieved, the modest sample size contributed to a relatively wide 95\% CI, underscoring the need for independent validation in a larger study; however, if similar performance was to be validated it would suggest that $30 \%$ of men suspicious for cancer after an initial negative biopsy could potentially be spared a repeat biopsy (31\% of men with a negative test for APC methylation $\times 96 \% \mathrm{NPV}$ ). This is the first prospective study in a defined clinical cohort with rigorous inclusion criteria to evaluate the potential usefulness of DNA methylation markers to predict outcome on repeat biopsy in this clinically important cohort.

We are aware of only one previous study that evaluated methylation markers in initial negative biopsies and compared them with histology in repeat biopsies. That study used archival samples and found 52-84\% sensitivity for detection of cancer on subsequent biopsies; NPV was not reported [18]. Few studies of promoter hypermethylation in prostate cancer have used DNA isolated from diagnostic biopsy tissue [19-22]; two have detected GSTP1 hypermethylation in histologically benign biopsies [21,22], and none have evaluated APC in benign biopsies.

In the present study, 21/86 (24\%) men with an initial negative biopsy had cancer detected in a 12-core repeat biopsy. Because NPV and PPV vary with cancer prevalence, unlike sensitivity and specificity, it is reassuring that the prostate cancer rate in our study overall, and within subgroups is consistent with values reported for similar populations

$[5,7,8,14,23,24]$. Among these men with cancer, APC was hypermethylated in 95\%, 
compared with GSTP1 in 43\%; rates similar to the other study on initial negative biopsies [18]. GSTP1 sensitivity is lower than that observed in previous studies that were based on histologically positive prostatectomy specimens. Because we evaluated methylation in biopsy specimens without histological evidence of cancer, it is possible that the initial biopsy either missed cancer that was subsequently detected on re-biopsy, or contained too few malignant cells to produce detectable morphological change. Conversely, GSTP1 has a higher specificity than APC, suggesting that methods be sought to exploit the advantages of both markers.

There are several clinically relevant findings from the present study. First, APC achieved high NPV while maintaining a low false-negative rate of 5\% (1/21), and high sensitivity of $95 \%$. APC also exhibited higher sensitivity and NPV then \%fPSA in the subset of participants with available data on PSA. Second, the difference in methylation ratios between cancer and histologically negative repeat biopsy was largest among the subgroup of men with HGPIN on initial biopsy, for both GSTP1 and APC (Table 1B). Because the clinical significance of HGPIN on biopsy remains unclear [14], methylation markers may provide additional tools for studying HGPIN. Third, methylation markers exhibited the lowest specificity in men with HGPIN or ASAP on initial biopsy. However, the cancer detection rate on second, and subsequent re-biopsy after initial biopsy diagnosis of HGPIN or ASAP is 35-50\% [6-9], suggesting that the rate of methylation false-positives could decrease with additional biopsies.

Notably, we observed that among participants with cancer, histologically negative initial biopsies frequently exhibited APC methylation alone, whereas GSTP1 methylation occurred only in those that also had APC methylation. Also, among participants with cancer, APC methylation ratios were significantly greater for those who also had GSTP1 methylation than for those with APC methylation alone. These findings are consistent with a gene methylation field effect where epigenetic changes in tissue adjacent to cancer foci precede detectable morphological alterations. Three recent studies observed potential field effects in prostatectomy specimens where APC (but not GSTP1) was hypermethylated in histologically benign areas distant from cancer or HGPIN $[15,25,26]$. A field effect occurring more frequently for APC than for GSTP1 is also consistent with higher NPV for APC. Alternatively, APC may be altered earlier in carcinogenesis than GSTP1, as in a recent study where hypermethylation of GSTP1 occurred in a coordinate fashion after hypermethylation of APC, RAR $\beta 2$ and RASSF1A [27].

Several potential limitations of the present study should be considered. First, among 122 men who met eligibility criteria $36(30 \%)$ could not be included in the analysis, primarily because of insufficient tissue or lack of available slides from initial biopsy. It is possible that their exclusion could create a selection bias against smaller tumours. However, those excluded did not differ significantly from the 86 analysed participants with respect to age, body mass index, DRE, PSA, \% fPSA, or PSA density, so selection bias is unlikely. Lack of tissue from some patients is more likely to represent the real constraints of accessing research specimens from fragile biopsy material remaining after clinical diagnostic evaluation. Second, a subject could be eligible with up to $33 \%$ of initial biopsy cores nonevaluable for methylation. This increases the likelihood of false-negatives, so the NPV of the biomarkers may actually be underestimated. This reflects the real-world clinical setting of the present study. The need for research tissue specimens was not anticipated when these clinically mandated initial biopsies were performed. If a methylation assay were to be translated to the clinic, greater care could be taken to safeguard tissue from the initial biopsy, or to develop assay methods requiring $<50 \mu$ of the FFPE block. Third, the threshold for APC methylation was determined post hoc based on the optimum value from the ROC curve, because we lacked sufficient previous APC data, generated using the ABI singlex 
PCR platform, to define an a priori theshold. Use of optimum thesholds can overstate diagnostic biomarker performance and so warrant cautious interpretation until independently validated [28]. However, threshold values in this range had been used previously [29]. Furthermore, we varied APC thresholds from 2.0 (optimum theshold) to 5.5 and still achieved NPV $\searrow 0.85$ and se $\searrow 0.71$ over that entire range. The number of false-positives was high with our APC threshold, as has been seen in other studies [18]. However, our objective was to achieve high NPV as a potential method of excluding re-biopsy. Given that re-biopsy would normally be performed for all men in the clinical scenario of our study, the biomarker false-positive rate is less important for this objective. Fourth, only $6 \%$ of men had $\geq 12$ cores on the initial biopsy. Our criterion was a minimum of sextant biopsy. It is possible that the initial biopsy would have fewer false-negatives if all men underwent 12-core biopsy.

However, the rate of cancer detection on initial biopsy (24\%) is similar to other clinical series, suggesting that significant undersampling relative to a 12-core sampling scheme is unlikely. Finally, data on other potential predictors, e.g. PSA, \%fPSA and prostate volume, were only available for 40-68\% of the original cohort, limiting our ability to evaluate the independent predictive value of APC or GSTP1. Nevertheless, all men had undergone an initial biopsy, so they had already exceeded a threshold of suspicion for cancer. Furthermore, these variables have not been shown to be good predictors of repeat biopsy outcome [30,31].

We showed that APC exhibits very high NPV in men with initial negative biopsy but high suspicion for cancer. Our results suggest that methylation markers could have the potential to avoid up to $30 \%$ of re-biopsies after an initial negative biopsy. Validation in a larger independent study is needed to determine whether APC methylation has sufficient precision to motivate a clinical trial. Future studies should also consider testing APC in panels with RAR $\beta 2$ and RASSF1A, which are also methylated in most prostate cancers and may demonstrate a field effect that enhances detection of cancer missed by histopathology.

\section{Supplementary Material}

Refer to Web version on PubMed Central for supplementary material.

\section{Acknowledgments}

Robin Gurganus (Johns Hopkins), Janet Vargo (Ortho-Clinical Diagnostics), Tatiana Vener (Ortho-Clinical Diagnostics), Haiying Wang (Ortho-Clinical Diagnostics), Abhijit Mazumder (Ortho-Clinical Diagnostics).

DISCLOSURES Funding to conduct the study was provided by Veridex, LLC (now MDx Health). Alan W. Partin was a study investigator funded by Veridex, LLC.

FUNDING B.J.T. and A.W.P. were supported in part by funding from the National Cancer Institute, grant Nos. P50-CA58236 (SPORE in Prostate Cancer) and U01-CA86323 (Early Detection Research Network)

\section{Abbreviations}

$\begin{array}{ll}\text { GSTP1 } & \text { glutathione-S-transferase P1 } \\ \text { APC } & \text { adenomatous polyposis coli } \\ \text { NPV } & \text { negative predictive value } \\ \text { \%fPSA } & \text { per cent free PSA } \\ \text { HGPIN } & \text { high-grade prostatic intraepithelial neoplasia } \\ \text { ASAP } & \text { atypical small acinar proliferation }\end{array}$



Ct
cycle threshold
PPV
positive predictive value
ROC
receiver operating characteristic

\section{REFERENCES}

1. Stamey TA, Caldwell M, McNeal JE, et al. The prostate specific antigen era in the United States is over for prostate cancer: What happened in the last 20 years? J Urol. 2004; 172:1297-1301. [PubMed: 15371827]

2. Roddam AW, Duffy MJ, Hamdy FC, et al. Use of prostate-specific antigen (PSA) isoforms for the detection of prostate cancer in men with a PSA level of 2-10 ng/ml: systematic review and metaanalysis. Eur Urol. 2006; 48:386-399. [PubMed: 15982797]

3. Ravery V, Goldblatt L, Royer B, et al. Extensive biopsy protocol improves the detection rate of prostate cancer. J Urol. 2000; 164:393-396. [PubMed: 10893593]

4. Babaian RJ, Toi A, Kamoi K, et al. A comparative analysis of sextant and an extended 11-core multisite directed biopsy strategy. J Urol. 2000; 163:152-157. [PubMed: 10604335]

5. Djavan B, Zlotta A, Remzi M, et al. Optimal predictors of prostate cancer on repeat prostate biopsy: a prospective study of 1,051 men. J Urol. 2000; 163:1144-1148. [PubMed: 10737484]

6. O'Dowd GJ, Miller MC, Orozco R, et al. Analysis of repeated biopsy results within 1 year after a noncancer diagnosis. Urology. 2000; 55:553-559. [PubMed: 10736500]

7. Lopez-Corona E, Ohori M, Scardino PT, et al. A nomogram for predicting a positive repeat biopsy in patients with a previous negative biopsy session. J Urol. 2003; 170:1184-1188. [PubMed: 14501721]

8. Singh H, Canto EI, Shariat SF, et al. Predictors of prostate cancer after initial negative systematic 12 core biopsy. J Urol. 2004; 171:1850-1854. [PubMed: 15076292]

9. Eggener SE, Roehl KA, Catalona WJ. Predictors of subsequent prostate cancer in men with a prostate specific antigen of 2.6 to $4.0 \mathrm{ng} / \mathrm{ml}$ and an initially negative biopsy. J Urol. 2005; 174:500 504. [PubMed: 16006880]

10. Keetch DW, Catalona WJ, Smith DS. Serial prostatic biopsies in men with persistently elevated serum prostate specific antigen values. J Urol. 1994; 151:1571-1574. [PubMed: 7514690]

11. Maruyama R, Toyooka S, Toyooka KO, et al. Aberrant promoter methylation profile of prostate cancers and its relationship to clinicopathological features. Clin Cancer Res. 2002; 8:514-519. [PubMed: 11839671]

12. Yegnasubramanian S, Kowalski J, Gonzalgo ML, et al. Hypermethylation of CpG islands in primary and metastatic human prostate cancer. Cancer Res. 2004; 64:1975-1986. [PubMed: 15026333]

13. Harden SV, Sanderson H, Goodman SN, et al. Quantitative GSTP1 methylation and the detection of prostate adenocarcinoma in sextant biopsies. J Natl Cancer Inst. 2003; 95:1634-1637. [PubMed: 14600096]

14. Epstein JI, Herawi M. Prostate needle biopsies containing prostatic intraepithelial neoplasia or atypical foci suspicious for carcinoma: implications for patient care. J Urol. 2006; 175:820-834. [PubMed: 16469560]

15. Mehrotra J, Varde S, Wang H, et al. Quantitative spatial resolution of the epigenetic field effect in prostate cancer. Prostate. 2008; 68:152-160. [PubMed: 18058812]

16. Moskowitz CS, Pepe MS. Comparing the predictive values of diagnostic tests: sample size and analysis for paired study designs. Clin Trials. 2006; 3:272-279. [PubMed: 16895044]

17. Bland JM, Altman DG. The use of transformation when comparing two means. BM. 1996; J 312:1153.

18. Troyer DA, Lucia MS, de Bruine AP, et al. Prostate cancer detected by methylated gene markers in histopathologically cancer-negative tissues from men with subsequent positive biopsies. Cancer Epidemiol Biomarkers Prev. 2009; 18:2717-22. [PubMed: 19755651] 
19. Harden SV, Guo Z, Epstein JI, et al. Quantitative GSTP1 methylation clearly distinguishes benign prostatic tissue and limited prostate adenocarcinoma. J Urol. 2003; 169:1138-42. [PubMed: 12576869]

20. Henrique R, Ribeiro FR, Fonseca D, et al. High promoter methylation levels of APC predict poor prognosis in sextant biopsies from prostate cancer patients. Clin Cancer Res. 2007; 13:6122-9. [PubMed: 17947477]

21. Rabiau N, Thiam MO, Satih S, et al. Methylation analysis of BRCA1, RASSF1, GSTP1 and EPHB2 promoters in prostate biopsies according to different degrees of malignancy. In Vivo. 2009; 23:387-91. [PubMed: 19454503]

22. Kwabi-Addo B, Wang S, Chung W, et al. Identification of differentially methylated genes in normal prostate tissues from African American and Caucasian men. Clin Cancer Res. 2010; 16(14):3539-47. [PubMed: 20606036]

23. Akhavan A, Keith JD, Bastacky SI, et al. The proportion of cores with high-grade prostatic intraepithelial neoplasia on extended-pattern needle biopsy is significantly associated with prostate cancer on site-directed repeat biopsy. BJU Intl. 2007; 99:765-769.

24. Girasole CR, Cookson MS, Putzi MJ, et al. Significance of atypical and suspicious small acinar proliferations, and high grade prostatic intraepithelial neoplasia on prostate biopsy: implications for cancer detection and biopsy strategy. J Urol. 2006; 175:929-933. [PubMed: 16469583]

25. Henrique R, Jerónimo C, Teixeira MR, et al. Epigenetic heterogeneity of high-grade prostatic intraepithelial neoplasia: clues for clonal progression in prostate carcinogenesis. Mol Cancer Res. 2006; 4:1-8. [PubMed: 16446401]

26. Steiner I, Jung K, Schatz P, et al. Gene promoter methylation and its potential relevance in early prostate cancer diagnosis. Pathobiology. 2010; 77:260-266. [PubMed: 21116117]

27. Florl AR, Steinhoff C, Müller M, et al. Coordinate hypermethylation at specific genes in prostate carcinoma precedes LINE-1 hypomethylation. Br J Cancer. 2004; 91:985-94. [PubMed: $15292941]$

28. Perkins NJ, Schisterman EF. The inconsistency of "optimal" cutpoints obtained using two criteria based on the receiver operating characteristic curve. Am J Epidemiol. 2006; 163:670-675. [PubMed: 16410346]

29. Jeronimo C, Henrique R, Hoque MO, et al. A quantitative promoter methylation profile of prostate cancer. Clin Cancer Res. 2004; 10:8472-8478. [PubMed: 15623627]

30. Ploussard G, Haese A, Van Poppel H, et al. The prostate cancer gene 3 (PCA3) urine test in men with previous negative biopsies: does free-to-total prostate-specific antigen ratio influence the performance of the PCA3 score in predicting positive biopsies? BJU Int. 2010; 106:1143-7. [PubMed: 20230386]

31. Shimbo M, Tomioka S, Sasaki M, et al. PSA doubling time as a predictive factor on repeat biopsy for detection of prostate cancer. Jpn J Clin Oncol. 2009; 39:727-31. [PubMed: 19674994] 


\section{What's known on the subject?}

Hypermethylation of genes such as glutathione-S-transferase P1 (GSTP1) and adenomatous polyposis coli (APC) occurs with high frequency in prostate tumour tissue but is much less common in the benign prostate; however, the potential value of gene methylation biomarkers as an adjunct to biopsy histopathology has had little study.

\section{What does the study add?}

When measured in histologically benign prostate biopsy tissue, APC gene hypermethylation was found to have high negative predictive value and high sensitivity. GSTP1 hypermethylation was found to have lower performance than APC. 


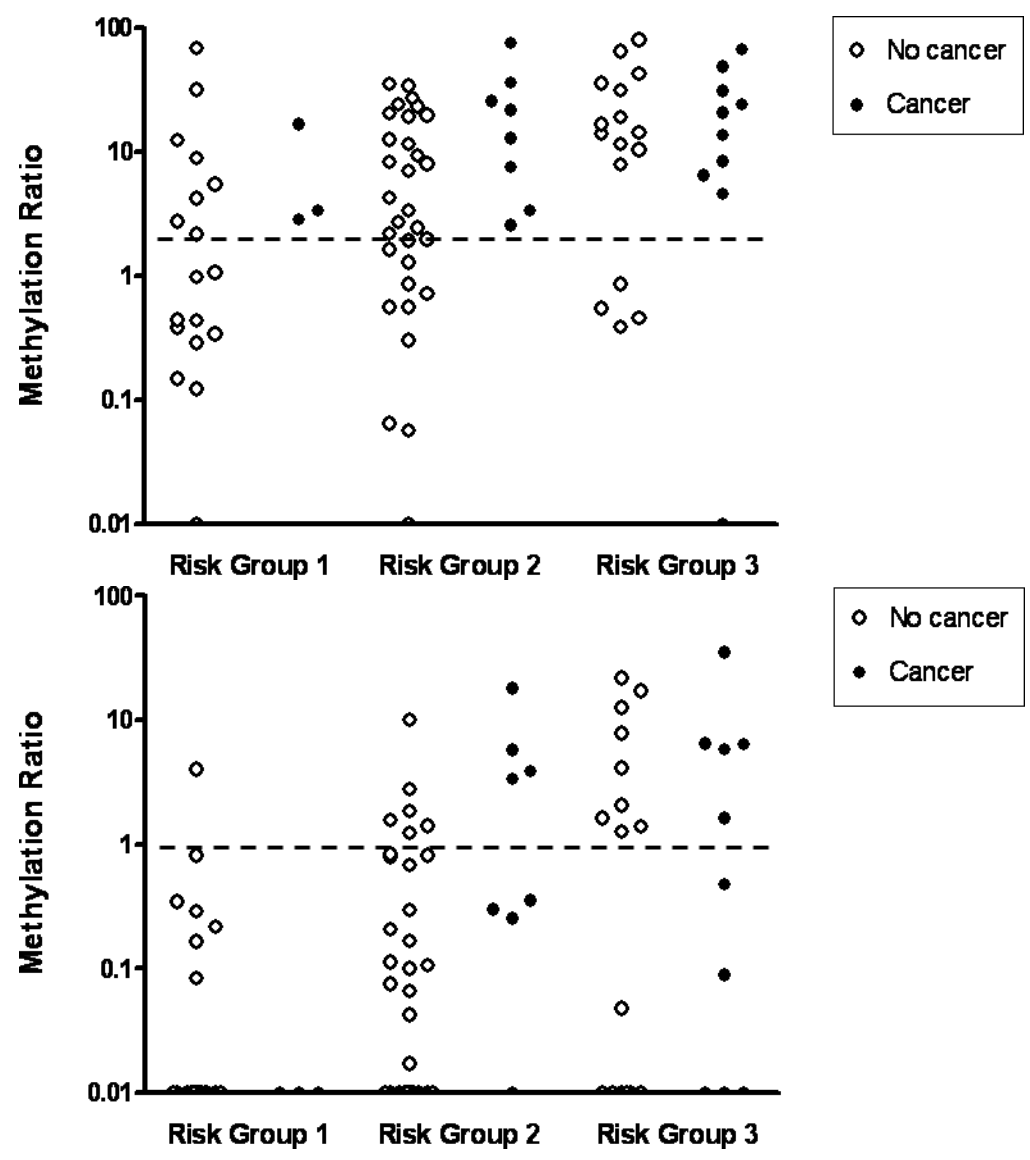

Fig. 1.

Methylation ratios by repeat biopsy histology (no cancer, cancer) for Groups 1, 2, and 3. A, APC methylation ratios, and B, GSTP1 methylation ratios. Dotted line indicates the threshold value for a positive test. Note: observations with a methylation ratio of 0.0 are shown on the graph as 0.01 owing to use of a logarithmic scale. Because the resolution of plotting symbols for points on the $\mathrm{X}$-axis makes it difficult to discern non-cancers (open circles) and cancers (solid circles), all points toward the left side within a risk group are noncancers, and points toward the right side within a risk group are cancers. 
Table 1A

Characteristics of study subjects by risk group: clinical and demographic characteristics

\begin{tabular}{|c|c|c|c|c|}
\hline Characteristic ${ }^{*}$ & & & & \\
\hline & Group 1, & Group 2, $n=39$ & Group 3, $n=26$ & $p$ \\
\hline Age, mean & 59.0 & 61.3 & 61.3 & 0.397 \\
\hline \multicolumn{5}{|l|}{ Race, $n(\%)$} \\
\hline White & $16(80.0)$ & $30(76.0)$ & $22(84.6)$ & \\
\hline Black & $3(15.0)$ & $8(20.5)$ & $2(7.7)$ & \\
\hline Other & $1(5.0)$ & $1(2.6)$ & $2(7.7)$ & 0.616 \\
\hline \multicolumn{5}{|l|}{ DRE, $n(\%)$} \\
\hline Normal/BPH & $18(85.7)$ & $31(83.8)$ & $22(84.6)$ & \\
\hline Suspicious & $3(14.3)$ & $6(16.2)$ & $4(15.4)$ & 0.981 \\
\hline Mean PSA level, ng/mL (n) & $8.2(17)$ & $6.6(33)$ & $5.3(15)$ & 0.235 \\
\hline Mean prostate volume, $\mathrm{cc}(n)$ & $39.2(18)$ & $45.1(28)$ & $36.9(10)$ & 0.425 \\
\hline PSA density, mean $\mathrm{ng} / \mathrm{mL} / \mathrm{cc}(n)$ & $0.25(14)$ & $0.16(26)$ & $0.14(12)$ & 0.078 \\
\hline \multicolumn{5}{|l|}{ Repeat biopsy outcome, $n(\%)$} \\
\hline No cancer & $18(85.7)$ & $31(79.5)$ & $16(61.5)$ & 0.981 \\
\hline Cancer & $3(14.3)$ & $8(20.5)$ & $10(38.5)$ & \\
\hline
\end{tabular}

* Characteristics were determined at the time of the initial biopsy, with the exception of cancer status, which was based on the second biopsy histology. 


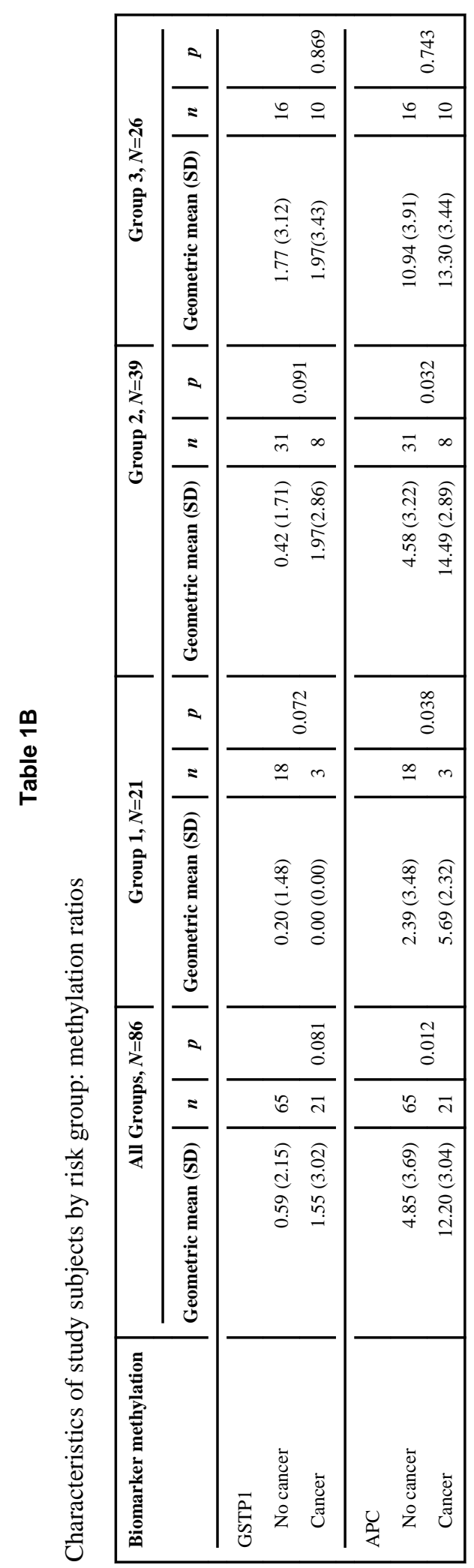

BJU Int. Author manuscript; available in PMC 2013 July 01. 


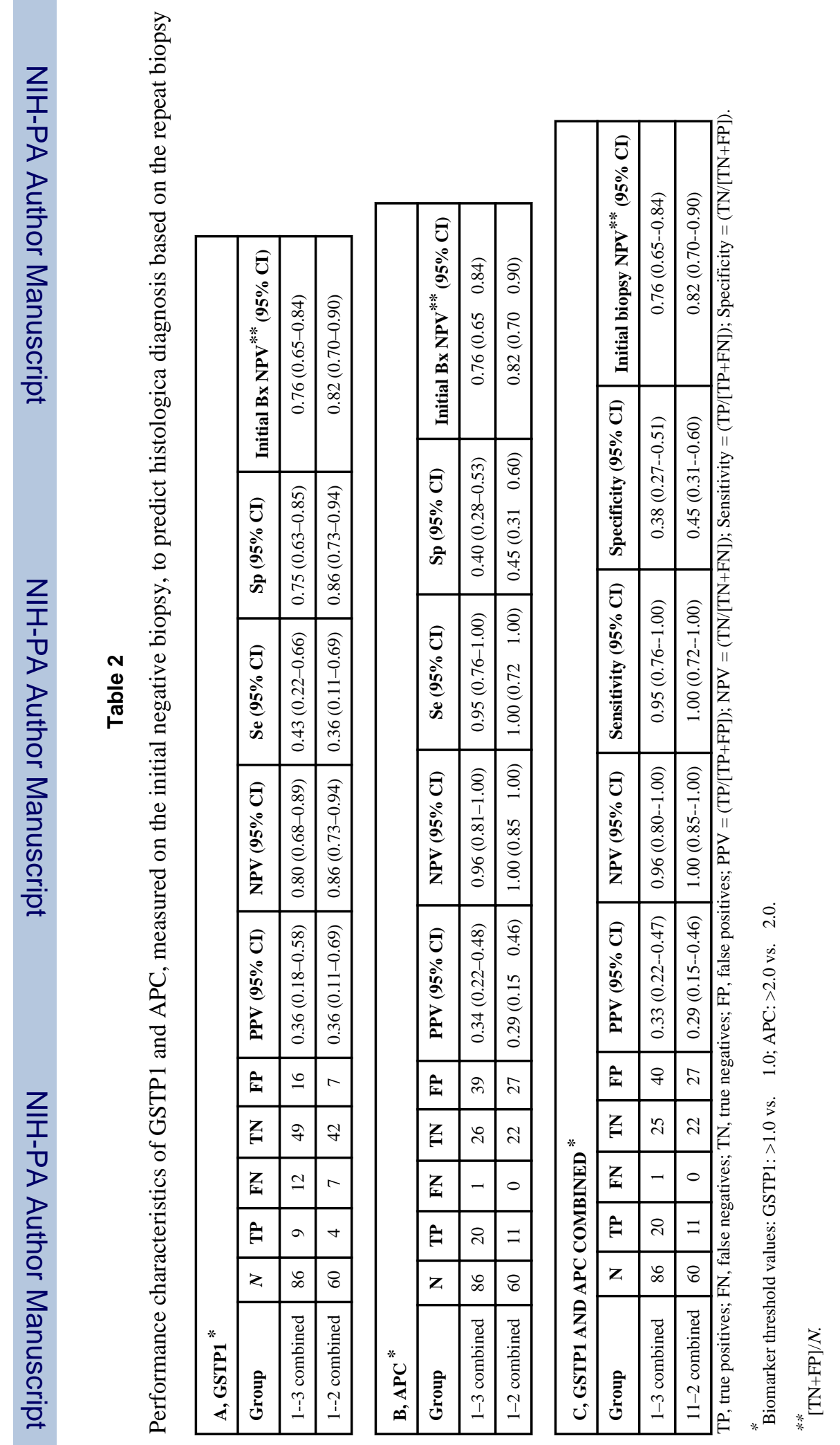

BJU Int. Author manuscript; available in PMC 2013 July 01. 